\title{
An analysis of outcomes of emergency physician/department-based thrombolysis for stroke
}

\author{
A P Volans
}

\section{Correspondence to} A P Volans, Emergency Department, Scarborough Acute Trust, Woodlands Drive, Scarborough, North Yorkshire Y012 60L, UK; andrew. volans@acute.sney.nhs.uk

Accepted 11 June 2011 Published Online First 21 September 2011

\begin{abstract}
Background Stroke thrombolysis is strongly supported as an effective therapy for selected cases of early stroke. The absence of $24 \mathrm{~h}$ stroke specialists in district general hospitals (DGHs) has led to the suggestion that regional hyper-acute stroke centres should be developed. This paper describes a cooperative model that uses the skills already present in a DGH to deliver a thrombolysis service initiated in the emergency department by the emergency physicians, and describes the outcomes of that service in comparison with the SITS-MOST trial.

Method The outcomes of all stroke patients thrombolysed at Scarborough DGH from 2004 to January 2009 were reviewed. Outcome was defined using a three-part scale. Data at Scarborough DGH were compared with data from the SITS-MOST European-wide study of stroke thrombolysis.
\end{abstract}

Results Data were available for 98 of 110 patients thrombolysed during the study period. Fifty (51\%) had a good outcome, seven (8\%) had partial resolution of their symptoms, and 41 (42\%) showed no improvement or deterioration. These outcomes were comparable to those in the European database.

Conclusion Stroke thrombolysis can be effectively delivered in a non-specialist (a non-hyper-acute stroke centre) DGH in the UK. An audit of cases completed describes complications seen.

\section{INTRODUCTION}

For many years, stroke was a condition that was primarily diagnosed by emergency physicians, but frustratingly few effective interventions were available in the emergency department (ED) to reduce the burden of this disease. Since 2007, intravenous thrombolysis has been recommended for the treatment of ischaemic stroke initially within $3 \mathrm{~h}$ of symptom onset, ${ }^{1-6}$ and more recently within $4 \frac{1}{2} \mathrm{~h}$ of symptom onset. ${ }^{7}$ The need for timely intervention in stroke has led to the redesign and development of services for stroke patients across the country.

A particular difficulty in the delivery of thrombolysis has been the reliance on non-ED physicians and services to make decisions in a timely manner. Such specialists are neither geographically nor chronologically available to many EDs in the UK, which receive patients $24 \mathrm{~h}$ a day. ${ }^{8}$ In many areas this has led to the development of stroke centres and/or pathways, with patients moving to the specialist centres for management. This is often over considerable distances and results in an increasing number of secondary transfers. An alternative model is for stroke and emergency medicine services to work collaboratively to provide thrombolysis at the location of the patient. ${ }^{8-11}$
Scarborough has adopted a collaborative approach that aims to deliver timely and appropriate thrombolysis for a population of stroke victims presenting to a district general hospital (DGH). The aim of this paper is to describe the service and to describe outcomes for stroke thrombolysis in a DGH setting.

\section{CONTEXT}

Scarborough Acute Trust is a geographically isolated, 320-bed DGH that lies 50 miles (90 min ambulance road time) from the next nearest hospitals. The hospital serves a population that varies with the tourist season from 220000 to $\sim 350000$ and has an ED census of 42000 patients at the DGH and supports a further 25000 minor cases seen in three peripheral minor injuries units. CT scanning is available $24 \mathrm{~h}$ a day. The hospital has a coronary care unit and an active multi-disciplinary stroke rehabilitation unit led by a general physician with a special interest in neurovascular medicine. Scarborough introduced stroke thrombolysis in August 2004, initially under the auspices of the Safe Implementation of Thrombolysis in Stroke-Monitoring Study (SITS-MOST) ${ }^{12}$ until the pathway had become established and the initial SITS-MOST trial closed (figure 1).

\section{METHODS}

The records of the research stroke nurse were reviewed to identify all patients treated by thrombolysis, with cross-checks with the admission registers of the coronary care unit and stroke unit.

The patient data and outcome data for the first 40 patients presenting to the ED at Scarborough were entered into the SITS-MOST registry, and the national and international comparisons are reported here. This database collects demographic, physiological, clinical and radiological data at presentation and records initial stroke severity using the NIH Stroke Scale (NIHSS) score and determines outcome at 7 days using a Global Score and at 3 months using the Rankin Scale. ${ }^{13}$

Early outcome data from Scarborough as compared with the SITS-MOST ${ }^{12}$ database is described using numbers and percentages. Formal statistical analysis was not attempted because of the relatively small numbers of patients in Scarborough's series. The SITS-MOST trial reported in 2008. ${ }^{12}$

The primary aim of this paper is to describe the outcomes and complications seen in the treated cohort.

For presentation of the local results of stroke outcome, patients were categorised at discharge using the following three pragmatic categories. 


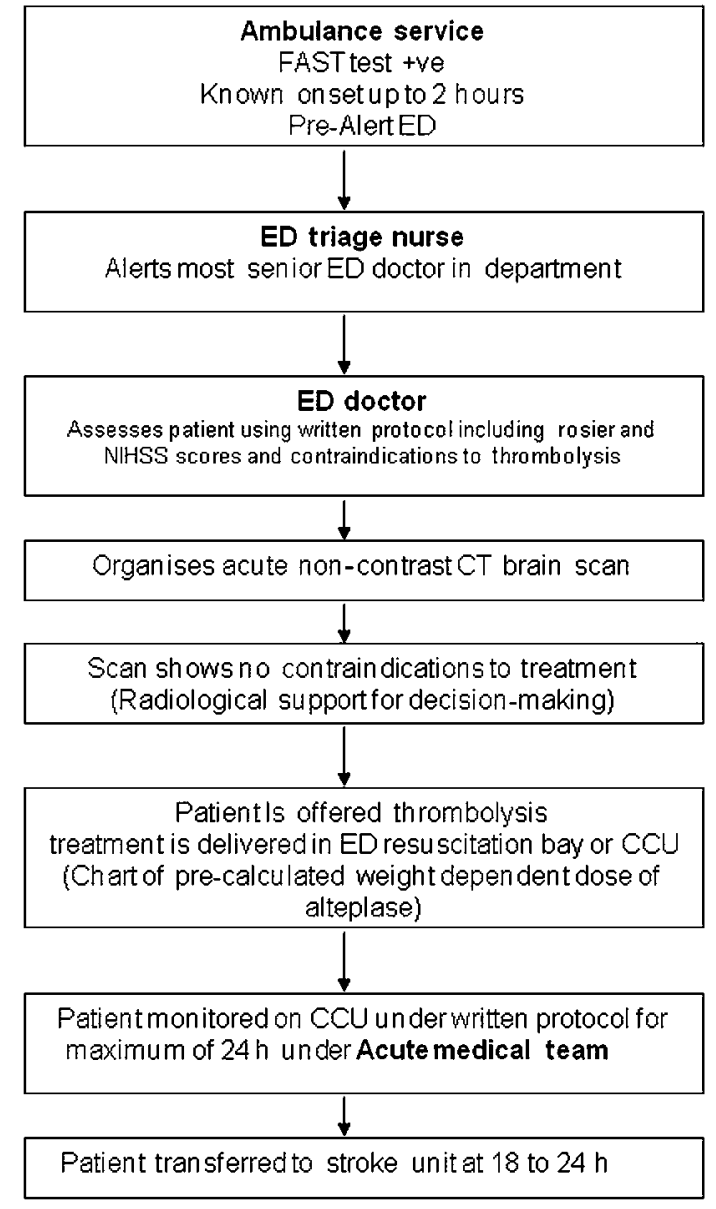

Figure 1 The Scarborough thrombolysis model of care. CCU, coronary care unit; ED, emergency department.

1. Patients who showed improvement in multiple areas of neurological loss, such as speech, power or sensory loss were rated as 'good responders'.

2. Patients who showed improvement in one area only-for example, speech or power-were rated as 'partial responders'.

3. Patients who showed no sign of improvement in any area of loss were rated as 'poor responders'.

Complications related to treatment (eg, significant intracerebral bleeding) were obtained from the patient notes. Patient mortality was determined at 6 months after presentation.

\section{RESULTS}

Between 2004 and early January 2009, Scarborough DGH treated 110 cases of stroke with thrombolysis. Clinical records were available for analysis in 98 cases.

The average age of the patients was 69 (range 38-91), with 54 men and 44 women. Seventy-nine patients were treated through the ED-based pathway, with an average time to treatment for the ED group of $2.7 \mathrm{~h}$ and a cohort 6-month mortality of $15 \%$. Nineteen cases from the wards were treated, having been admitted for a number of other diagnoses including transient ischaemic attack. The time to treatment in the ward group was $3.4 \mathrm{~h}$, with a cohort 3-month mortality of $12 \%$.

Table 1 shows the CT, Global Score at 7 days and Rankin outcomes for patients at Scarborough and compares these with the data for UK and European data.

Early deaths occurred in seven of 98 patients, all in the nonresponding group. Five patients died on day 1 and in four of
Table 1 SITS-MOST entries (first 40 cases)

\begin{tabular}{lcll}
\hline & $\begin{array}{l}\text { SGH } \\
\text { (\% of cases) }\end{array}$ & $\begin{array}{l}\text { UK data } \\
\text { (\% of cases) }\end{array}$ & $\begin{array}{l}\text { Total European } \\
\text { data (\%) }\end{array}$ \\
\hline NIHSS score & & & \\
$0-7$ & $25(10 / 40)$ & 15 & 21 \\
$8-14$ & $22(9 / 40)$ & 32 & 36 \\
15+ & $50(20 / 40)$ & 51 & 39 \\
CT findings* & & & \\
$\quad$ Baseline scan: no infarct & $97(39 / 40)$ & 58 & 77 \\
Follow-up scan: no infarct & $20(8 / 40)$ & 14 & 23 \\
$\quad$ Follow-up scan: infarct & $77.5(31 / 40)$ & 85 & 75 \\
Global score at 7 days & & & \\
Much better & $45(18 / 40)$ & 39 & 41 \\
Better & $15(6 / 40)$ & 31 & 30 \\
Unchanged & $30(12 / 40)$ & 13 & 15 \\
Worse & $3(1 / 40)$ & 4 & 5 \\
Dead & $6(2 / 40)$ & 9 & 7 \\
Rankin score at 3 months & & & \\
0 \& 1 & $27.5(11 / 40)$ & 22 & 34 \\
2 \& 3 & $24(10 / 40)$ & 36 & 27 \\
4 \& 5 & $20(8 / 40)$ & 18 & 19 \\
6 (death) & $28(11 / 40)$ & 21 & 14
\end{tabular}

${ }^{*}$ CT scans were scored using SITS-MOST criteria.

NIHSS, NIH Stroke Scale.

these, a CT scan showed massive cerebral oedema. The 5th patient died before a repeat CT could be performed and might represent a post-thrombolysis intracerebral haemorrhage. The 5 th oedema death was 5 days after stroke onset.

Late deaths were associated with failure to rehabilitate and had common complications of stroke recorded in the notes such as aspiration pneumonia, new strokes and pulmonary emboli.

During 2008, there were 451 cases of stroke admitted acutely to the hospital as reported in the Hospital Activity Statistics. During this year, 49 (11\%) cases were treated with the integrated pathway.

\section{DISCUSSION}

Our study shows that thrombolysis for stroke can be achieved in a DGH. Our patient selection and patient outcomes are similar to those achieved across the UK and across Europe. ${ }^{12}$

Using our pathway, we treated $11 \%$ of patients presenting with acute ischaemic stroke during the last year of full data collection for this paper, which is similar to the predicted model for UK departments.

Our findings are important to the wider emergency medicine audience for several reasons. First we have demonstrated the feasibility of providing a thrombolysis service outside of a hyperacute stroke centre. Although we developed this service because of the geographical location of Scarborough, the underlying principle that therapy should be available at the patient's location, as opposed to the patient having to be transferred to where the therapy is, is supported by our findings. In this respect, thrombolysis for stroke is perhaps similar to the initial strategies for thrombolysis in acute myocardial infarction. When initially introduced, thrombolysis for acute myocardial infarction was restricted to specialist centres and under the guidance of cardiologists. With time and experience, it became clear that a treatment that is portable is best given at the point of need, thereby reducing the delay inherent in moving the patient to the treatment. Our experience in Scarborough clearly shows that this is achievable (table 2).

Second, we have demonstrated that the clinical outcomes in a DGH population are similar to those in a European-wide 
Table 2 Outcomes: mortality and neurological change of 98 recorded cases

\begin{tabular}{ll}
\hline Neurological change in first 7 days & \\
\hline Good (improvement of all areas of neurological abnormality) & $50(51 \%)$ \\
Partial (improvement in one area of neurological abnormality) & $7(8 \%)$ \\
No improvement or deterioration & $41(42 \%)$ \\
Mortality & \\
Case mortality all strokes admitted (2008) & $30 \%$ \\
Case mortality (thrombolysed cohort, all years) & $14 / 98,14 \%$ \\
Cohort mortality $<7$ days & $7 / 98,7 \%$ \\
Cohort mortality $>7$ days $<6$ months & $8 / 98,8 \%$ \\
\hline
\end{tabular}

cohort. Although the numbers of patients in our study is relatively small, our results suggest that outcomes are likely to be comparable. Unfortunately, the number of patients in this study is too small to statistically show equivalence, and indeed it is unlikely that a single centre such as ours will ever acquire a study population large enough to do this. Further work and experience from other units may confirm this, as it eventually did for thrombolysis for myocardial infarction (table 3).

The Scarborough model depends on cooperative working between different units that already have the basic skills required to perform thrombolysis in stroke patients. Although the majority of strokes seen in the ED are 'barn door', the use of objective scoring systems (NIHSS) and the clinical availability of colleagues in neurology and stroke care have supported decision making in difficult cases. Similarly, an enthusiastic and supportive radiology service has been essential in the early differentiation of cerebral pathology. Although the majority of CT scans are interpreted by senior ED or stroke physicians, timely radiology support is essential in some cases.

Support and enthusiasm for the service has been reinforced by inviting patients who had made significant improvements to visit the emergency and radiological departments. This has rapidly developed a system-wide 'ownership' of the service.

Most patients presented during 'extended office hours', with the bulk of our cases being treated between 07:00 and midnight. Logically, in our semi-rural population, strokes occurring after this time will occur during sleep, and onset can therefore not be identified. Populations with a more extended lifestyle will probably require an overnight service as well.

A number of patients had adverse effects related to the treatment. Catastrophic intracerebral haemorrhage possibly occurred in one case where a poor responder deteriorated suddenly and died before a repeat CT scan could be performed. Five other patients suffered sudden deterioration and had CT findings of massive cerebral oedema. Bleeding from occult gastrointestinal lesions was seen in 10 cases in the early days after treatment, two of these cases from a previously unknown carcinoma. One case of angio-oedema and hypotension was seen (table 4).

Major complications are therefore not uncommon and we believe that this supports our practice of observing stroke patients receiving thrombolysis on the coronary care unit where nursing staff are experienced in the assessment and management of thrombolysis complications.

Table 3 Mortality by outcome group

\begin{tabular}{llll}
\hline & Overall & Early $(<7$ days $)$ & $\begin{array}{l}\text { Late }(\mathbf{7} \text { days }- \\
\mathbf{3} \text { months })\end{array}$ \\
\hline Good outcome & $1 / 50(2 \%)$ & 0 & $1(2 \%)$ \\
Partial outcome & $0 / 7$ & 0 & 0 \\
No improvement & $11 / 41(27 \%)$ & $7(17 \%)$ & $4(10 \%)$ \\
\hline
\end{tabular}

Table 4 Complications seen in the treated cohort (98 patients)

\begin{tabular}{llr}
\hline Outcome & Complication & No \\
\hline Good responders & Minor superficial bleeds (cannula sites) & 2 \\
Partial responders & & 0 \\
Poor responders & Gastrointestinal bleed & 10 \\
& Minor ICH changes & 7 \\
& Cerebral oedema & 5 \\
& Cardiac complications & 4 \\
& DVT & 3 \\
& Pulmonary embolus & 2 \\
& 2nd CVA within 1 month & 2 \\
& Possible major ICH & 1 \\
& Acute angio-oedema & 1 \\
& Minor superficial bleeds (cannula sites) & 1
\end{tabular}

CVA, cerebrovascular accident; DVT, deep vein thrombosis; ICH, intracranial haemorrhage.

The 3-month death rate among the first 40 cases reviewed was higher than in the SITS-MOST database. When this was recognised, the cases were reviewed, and it was found that the deaths were occurring in non-responding patients significantly over 80 years of age. This resulted in a tightening of selection procedures with a resulting fall in death rates in the later cohort. This finding reinforces the value of a national reporting strategy for new interventions.

Our study is clearly limited by being single centre and with a relatively small number of patients. However, our aim was to demonstrate the feasibility and achievability of performing stroke thrombolysis in a DGH setting. We believe that we have demonstrated this and suggest that our model of bringing thrombolysis to the patient, rather than the patient to thrombolysis, ${ }^{11}$ is one that could be successfully replicated elsewhere.

\section{CONCLUSIONS}

Thrombolysis for acute ischaemic stroke is achievable and safe within a DGH setting. A DGH-based thrombolysis service could potentially offer thrombolysis to $10 \%$ of patients presenting with an acute stroke. Thrombolysis should be available at the place where patients with acute ischaemic stroke present. Outcomes from DGH-based thrombolysis are comparable to those in the SITS-MOST database.

\section{Competing interests None.}

Contributors AP Volans performed the analysis and wrote the paper. John Patterson supplied the protocols and advice on content.

Provenance and peer review Not commissioned; externally peer reviewed.

\section{REFERENCES}

1. Swain S, Turner C, Tyrrell $P$, et al. Diagnosis and initial management of acute stroke and transient ischaemic attack: summary of NICE guidance. BMJ 2008;337:a786

2. Tyrrell P, Swain S, Rudd A; Acute Stroke \& TIA Guideline Development Group. NICE guideline on acute stroke and TIA: commentary. Heart 2009;95:843-5.

3. Tissue plasminogen activator for acute ischemic stroke. The National Institute of Neurological Disorders and Stroke rt-PA Stroke Study Group. N Engl J Med 1995;333:1581-7.

4. Wahlgren NG, Ahmed N, Davalos A, et al. Thrombolysis with alteplase for acute ischaemic stroke in the Safe Implementation of Thrombolysis in Stroke-Monitoring Study (SITS-MOST): an observational study. Lancet 2007;369:275-82.

5. National Institute for Health and Clinical Excellence. Diagnosis and initial management of acute stroke and transient ischaemic attack (TIA). CG68. London: National Institute for Health and Clinical Excellence, 2008.

6. Sandercock $\mathbf{P}$, Wardlaw J, Lindley R. "Where are we now with intravenous thrombolysis for acute ischaemic stroke"? Int J Stroke 2010;5:381-2.

7. Hacke W, Kaste M, Bluhmki E, et al. Thrombolysis with Alteplase 3 to 4.5 hours after Acute Ischaemic Stroke. N Engl J Med 2008;359:1317-29.

8. McCarron MO, Armstrong M, McCarron P. Potential for quality improvement of acute stroke management in a district general hospital. Emerg Med J 2008;25:270-4. 
9. Kobayashi A, Skwronska M, Litwin T, et al. Lack of experience of intravenous thrombolysis for acute ischaemic stroke does not influence the proportion of patients treated. Emerg Med J 2007;24:96-9.

10. Tveiten A, Mygland A, Ljostad U, et al. Intravenous thrombolysis for ischaemic stroke: short delays and high community-based treatment rates after organisational changes in a previously inexperienced centre. Emerg Med $\mathrm{J}$ 2009;26:324-7.
11. Semlicini A, Benetton V Macchini L et al. Intravenous thrombolysis in the emergency department for the treatment of acute stroke. Emerg Med J 2008;25:403-6.

12. Thrombolysis with alteplase for acute ischaemic stroke in the Safe Implementation of Thrombolysis in Stroke-Monitoring Study (SITS-MOST): an observational study. Stroke 2008;39:3316-22.

13. Rankin J. Cerebral vascular accidents in patients over the age of 60. Scott Med $J$ 1957:2:200-15.

\section{Images in emergency medicine}

\section{What lies beneath: an unusual case of tuberculous osteomyelitis}

A 28-year-old man attended the emergency department after a transient episode of unresponsiveness. Further questioning revealed weight loss over the preceding 2 months, accompanied

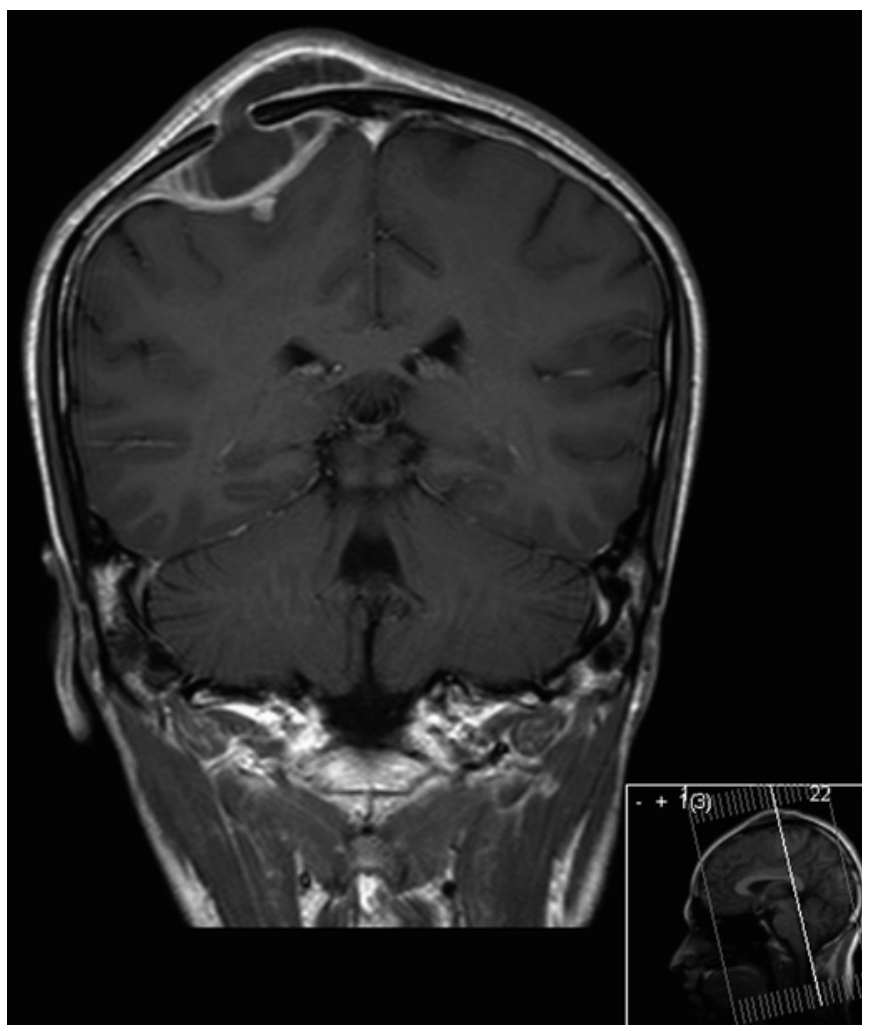

Figure 1 Coronal MRI (with contrast) section of the head showing an $8 \mathrm{~mm}$ skull defect through which the intra and extracranial components of the collection communicate. Signal abnormality is seen in the adjacent skull bone. by the emergence of a fluctuant 'bulge' overlying his right forehead. He complained of intermittent vomiting and sweating.

Examination revealed a noticeable $2 \mathrm{~cm} \times 3 \mathrm{~cm}$ fluctuant mass overlying his right forehead. Cranial nerve examination demonstrated an obvious lower motor neuron seventh cranial nerve deficit. Blood profiles were within normal parameters and HIV serology was negative. A chest radiograph revealed an illdefined right field apical opacification; however, subsequent cranial contrast CT demonstrated an enhancing mass within the right frontoparietal region with extensive vasogenic oedema. MRI confirmed an $8 \mathrm{~mm}$ right frontal bone defect, with adjacent reactive bone proliferation (figure 1). A diagnosis of secondary cerebral tuberculosis/tuberculous skull osteomyelitis was made, confirmed by PCR-positive sampling from the fluctuant mass. The patient made an uneventful recovery after combination therapy for tuberculosis. His facial palsy resolved within weeks. His skull defect was closed electively at a later date.

Our case demonstrates an unusual and unanticipated mixed presentation of tuberculosis. Importantly, thresholds for timely emergent radiographic review in apparently innocuous presentations ought to be assessed on a case-by-case basis.

\section{Marieke Bokhoven, Jonathan Costello}

Department of Emergency Medicine, Royal Free Hampstead NHS Trust, London, UK

Correspondence to Dr Marieke Bokhoven, Department of Emergency Medicine, Royal Free Hampstead NHS Trust, London NW3 6YA, UK; m.bokhoven@nhs.net

Contributors MB assessed the patient on admission to the emergency department $\mathrm{MB}$ and $\mathrm{JC}$ wrote the manuscript.

Competing interests None.

Patient consent Obtained.

Provenance and peer review Not commissioned; internally peer reviewed.

Accepted 28 April 2012

Published Online First 29 May 2011

Emerg Med J 2012;29:643. doi:10.1136/emermed-2012-201481 